TRABAJOS ORIGINALES

Rev Obstet Ginecol Venez. 2022; 82 (1): 21-32.

https://doi.org/10.51288/00820105

\title{
Ecografía transperineal intraparto en la progresión del trabajo de parto*
}

\author{
Génesis Jesmay Cárdenas Mendoza, ${ }^{1}$ (D) Mayrín María Martínez Pérez, ${ }^{1}$ (D) Shiuna Lo Huang, ${ }^{2}$ \\ Jesús Veroes. ${ }^{3}$
}

\section{RESUMEN}

Objetivo: Determinar la utilidad de la ecografia transperineal intraparto en la progresión del trabajo de parto en las pacientes ingresadas al Servicio de Sala de Partos de la Maternidad Concepción Palacios, en el periodo mayo-agosto de 2019.

Métodos: Estudio prospectivo, descriptivo y longitudinal que incluyó 98 pacientes. Se realizó ecografía transperineal cada dos horas durante el trabajo de parto, evaluando características del cuello uterino y cambios en la variedad de posición y descenso de la cabeza fetal, relacionándolos con los hallazgos clínicos.

Resultados: Se realizaron 219 ecografias. Al ingreso, la media ecográfica de la dilatación fue de 5,94 $\pm 1,70 \mathrm{~cm}$ y por tacto fue 5,96 $\pm 1,80 \mathrm{~cm}(p=0,717)$. A las dos horas, fue $8,14 \pm 1,92 \mathrm{~cm}$ y 8,15 $\pm 2,07 \mathrm{~cm}$, respectivamente $(p=0,849)$. Al tercer control, fue $8,35 \pm 1,70 \mathrm{~cm}$ por ecografía y por tacto fue $8,43 \pm 1,97(p=0,604)$. Respecto al borramiento, entre las 4 pacientes con grado 3 (el menor borramiento), 1 (25\%) tenía cuello borrado $40 \%$ - $50 \%$ y 3 (75\%), $60 \%$ - $70 \%$. Las $116(100 \%)$ pacientes diagnosticadas ecográficamente como grado 0 (el máximo de borramiento), tenían cuello borrado más de $80 \%$. Hubo asociación entre el plano de Hodge con la distancia cabeza-periné $(p=0,001)$ y con el ángulo de línea media ( $p=0,001)$. No hubo asociación con la distancia cabeza-sínfisis. La satisfacción fue de $100 \%$.

Conclusión: La ecografía transperineal intraparto es útil en la evaluación de la progresión del trabajo de parto.

Palabras clave: Ecografia transperineal, Ecografia intraparto, Trabajo de parto, Variedad de posición, Descenso cabeza fetal.

\section{Intrapartum transperineal ultrasound in the progression of labour} SUMMARY

Objective: To determine the usefulness of intrapartum transperineal ultrasound in the progression of labor in patients admitted to the Concepción Palacios Maternity Parting Room Service, in the period May-August 2019.

Methods: Prospective, descriptive and longitudinal study involving 98 patients. Transperineal ultrasound was performed every two hours during labor, evaluating cervical characteristics and changes in fetal head position and decline variety, linking them to clinical findings.

Results: 219 ultrasounds were performed. At the begin, the ultrasound mean of the dilation was $5.94 \times 1.70 \mathrm{~cm}$ and by clinic was $5.96 \times 1.80 \mathrm{~cm}(p-0.717)$. At two hours, it was $8.14 \times 1.92 \mathrm{~cm}$ and $8.15 \times 2.07 \mathrm{~cm}$, respectively (p-0.849). At the third control, it was $8.35 \times 1.70 \mathrm{~cm}$ per ultrasound and by clinic it was $8.43 \times 1.97$ (p.0.604). With regard to erasure, among the 4 patients with grade 3 (the least erasure), 1 (25\%) neck erased 40\%-50\% and 3 (75\%), 60\% - 70\%, 116 (100\%) patients ultrasound as grade 0 (the maximum erasure), had a neck erased by more than $80 \%$. There was an association between Hodge's plane with the head-to-perineum distance (p-0.001) and the mid-line angle (p-0.001). There was no association with head-symphysis distance. Satisfaction was 100\%.

Conclusion: Intrapartum transperineal ultrasound is useful in evaluating the progression of labor.

Keywords: Transperineal, Position variety, Fetal head descent.

${ }^{1}$ Especialistas en Obstetricia y Ginecología. ${ }^{2} E$ specialistas en Obstetricia y Ginecología, adjunto del Servicio de Prenatal, Maternidad Concepción Palacios. ${ }^{3}$ Especialista en Obstetricia y Ginecología y en Medicina Materno-Fetal. * Trabajo Especial de Grado que se presentó para optar al título de especialista en Obstetricia y Ginecología en la Universidad Central de Venezuela. Correo de correspondencia: genesiscardenas278@gmail.com

Forma de citar este artículo: Cárdenas GJ, Martínez MM, Lo Huang S, Veroes J. Ecografía transperineal intraparto en la progresión del trabajo de parto. Rev Obstet Ginecol Venez. 2022; 82(1): 21-32. https://doi. org/10.51288/00820105

\section{INTRODUCCIÓN}

El parto es el proceso fisiológico que pone fin al embarazo, por el cual se produce la salida desde el útero hacia el exterior a través del canal del parto, de un feto, vivo o muerto, seguido de la placenta y de las membranas ovulares (1). 
Toda mujer embarazada en trabajo de parto, espontáneo o por inducción del mismo, desea saber si va a tener un parto vaginal o necesitará un parto quirúrgico; la habilidad y juicio necesarios para decidir cuándo y cómo intervenir han sido factores definitorios en el arte de la obstetricia.

Tradicionalmente, la evaluación y el manejo del trabajo de parto se basan en los hallazgos clínicos a través del tacto vaginal, este siempre ha sido el estándar de oro mediante el cual se diagnostican las distocias del parto, como la detención del descenso. También se puede evaluar la maduración cervical y dilatación cervical y la posición de la cabeza fetal, antes y durante el trabajo de parto, basando las decisiones sobre el momento y el tipo de intervención según estos hallazgos clínicos. Sin embargo, el examen digital suele ser inexacto y subjetivo (2), principalmente en los casos donde existe la presencia de asinclitismo y caput succedaneum en los cuales se dificulta la palpación de las suturas y fontanelas, y que están frecuentemente asociados a parto obstruido.

A medida que ha evolucionado la obstetricia, se ha apreciado la implementación de diferentes técnicas para la evaluación, tanto del inicio del trabajo de parto como de su progresión, y una de estas técnicas es la realización de la ecografía transperineal intraparto, la cual parece ser más exacta a la hora de evaluar las distintas etapas del trabajo de parto y ha ayudado a complementar la evaluación tradicional digital (3), a través del estudio de diferentes medidas objetivas como lo son la variedad de posición, el ángulo de progresión, la dilatación del cuello uterino y el descenso de la cabeza fetal (3).

En la literatura médica se encuentran múltiples investigaciones relacionadas con el tema. Desde la medición de la dilatación cervical por ultrasonido 2D transperineal, considerada un método factible y preciso (2), la descripción de la posibilidad de medir el descenso de la cabeza fetal a través de la distancia desde el cráneo fetal hasta el perineo, así como la distancia entre la cabeza fetal y la sínfisis del pubis (3), y la determinación de que un ángulo de progresión mayor a $120^{\circ}$ se asocia a una probabilidad del $90 \%$ de tener un parto vaginal exitoso (4). Además, en mujeres en fase activa del trabajo de parto, se han utilizado los siguientes marcadores ecográficos: ángulo de progresión, distancia cabeza - perineo y distancia cabeza - sínfisis del pubis, y se ha comparado la ecografía transperineal con la evaluación digital. Estos son más precisos que el examen digital al evaluar el tipo de parto, sobre todo, si se altera el examen vaginal debido a la presencia de caput succedaneum (5). Se considera que la evaluación del ángulo de progresión hasta obtener la dilatación completa representa el mejor marcador ecográfico y ayuda en la predicción de prolongación de la segunda etapa del trabajo de parto, considerándolo un parámetro necesario a la hora de predecir un parto vaginal exitoso (6 - 8) y de evaluar la estación de la cabeza fetal, el descenso y la rotación $(9,10)$. Otros autores han descrito conclusiones similares, con relación a que la evaluación ecográfica del trabajo de parto posee una buena concordancia con los exámenes vaginales digitales $(11-14)$. Además, se considera que el examen de ultrasonido es más preciso y reproducible que el examen clínico en el diagnóstico de la posición de la cabeza fetal y la estación y en la predicción de la detención del parto, dando objetividad a esta evaluación (15 - 17).

Hasta el desarrollo del partograma, a mediados del siglo $\mathrm{XX}$, la evaluación del progreso del trabajo de parto, fue subjetiva y, a menudo, se supervisó de acuerdo con su duración. El partograma fue un avance crucial que permitió una evaluación objetiva del progreso del trabajo de parto mediante el trazado de la dilatación cervical y la estación fetal en función del tiempo y permitió que los trastornos del trabajo se definieran y clasificaran. El manejo del descenso prolongado, detención del descenso o falta de descenso, es uno de los problemas más difíciles de la obstetricia. De hecho, la habilidad y el juicio necesarios para decidir cuándo y cómo intervenir han sido factores definitorios en el 
arte de la obstetricia. Sería ideal si, antes del inicio del trabajo de parto o en sus primeras etapas, se pudiera predecir con exactitud quién tendrá un parto por vía vaginal sin complicaciones y quién necesitará un parto quirúrgico (abdominal o asistido) (17).

La guía práctica de la International Society of Ultrasound in Obstetrics and Gynecology (ISUOG) describe ampliamente y en detalle la técnica de medición de los diferentes parámetros (16). Esta técnica puede ser vía transabdominal (principalmente para determinar la posición de la cabeza y columna vertebral) o transperineal (principalmente para evaluar la estación y posición en estaciones bajas). Se utiliza una sonda convexa, similar a la empleada para determinar la biometría fetal y la evaluación anatómica. Se ha sugerido una serie de parámetros ecográficos con una alta coincidencia intra- e interobservador, los cuales se describen a continuación.

La evaluación de la posición de la cabeza fetal, mediante ecografía transabominal, con la paciente en decúbito supino, sin pujos activos y con la vejiga vacía. El transductor se coloca de forma transversa en la región suprapúbica del abdomen materno, visualizando la cabeza fetal; los puntos de referencia para determinar la variedad de posición son los siguientes: visualización de las órbitas (occipitoposterior), línea media cerebral (occipito-transversa) o cerebelo (occipito-anterior) $(15,17)$.

La determinación de la variedad de posición de la cabeza fetal, que se puede describir representando un círculo, como un reloj: las posiciones $\geq 02: 30$ horas y $\leq 03: 30$ horas, deben registrarse como occipital transversal izquierdo (OTI); las posiciones $\geq 08: 30$ horas y $\leq 09: 30$ horas, como occipital transversal derecho (OTD); las posiciones $>03: 30$ horas y $<08: 30$ horas deben registrarse como occipital posterior; las posiciones $>09: 30$ horas $\mathrm{y}<02: 30$ horas como occipucio anterior (16).
La evaluación de la estación de la cabeza fetal, mediante ecografía transperineal o translabial, con la paciente en posición decúbito supino, con las piernas flexionadas en las caderas y las rodillas en $45^{\circ}$ y $90^{\circ}$ respectivamente; es esencial que la vejiga esté vacía. El transductor abdominal se recubre con una funda de látex y gel ecográfico y se coloca entre los labios mayores, a nivel de la horquilla vulvar, en posición media sagital. En el corte obtenido se permite determinar las siguientes marcas anatómicas: a) articulación de la sínfisis del pubis, como una estructura ecogénica oblonga, irregular; idealmente se muestra en una posición horizontal; b) cráneo fetal, con tábula anterior y posterior; c) las espinas ciáticas no se ven por esta vía $(15,16)$.

En las imágenes transperineales en el plano medio sagital, se han propuesto varios parámetros que utilizan la sínfisis púbica como punto de referencia para las mediciones cuantitativas. Tres indican la estación principal directamente: el ángulo de progresión (AoP), también llamado ángulo de descenso; la distancia de progresión (DP) y la estación de la cabeza al ultrasonido transperineal.

El ángulo de progresión es un parámetro preciso y reproducible para la evaluación de la cabeza fetal. Es el ángulo que se forma entre el eje largo del hueso púbico y el borde más bajo del pubis, tangencial a la parte ósea más profunda del cráneo fetal (16). En la imagen sagital se dibuja una línea a través del eje longitudinal de la sínfisis del pubis y una segunda línea que se extiende desde la parte más inferior del pubis, tangencialmente al contorno del cráneo del feto y se realiza la medición del ángulo entre las líneas construidas. Se caracteriza por presentar una mínima variabilidad intra- e interobservador (17). Al comparar el ángulo de progresión y la estación fetal al ultrasonido transperineal, se evidencia que la estación cero de la cabeza fetal, corresponde a un ángulo de progresión de $116^{\circ}$ (16). Un ángulo de progresión mayor o igual a $120^{\circ}$ es un predictor excelente de parto vaginal 
(90\% de los casos); si existe un segundo período del parto prolongado pero el ángulo de progresión es mayor o igual de $120^{\circ}$, se dice que tiene opción a parto vaginal espontáneo (16).

La distancia de progresión, se define como la distancia mínima entre la línea infrapúbica y la presentación (16).

La estación sonográfica de la cabeza, expresa la estación principal en la escala utilizada convencionalmente para la evaluación palpatoria del progreso del trabajo de parto (planos de De Lee) e incorpora la curvatura del canal del parto. Requiere una evaluación de la dirección de la cabeza y la distancia entre el plano infrapúbico (que está a $3 \mathrm{~cm}$ por encima del plano isquiático) y la parte ósea más profunda que se presenta a lo largo de la línea de la dirección de la cabeza. Si bien es más complejo de medir (porque requiere mediciones de ángulo y distancia), se encontró que se correlaciona linealmente con el ángulo de progresión fácilmente medible: la relación entre estos dos parámetros permite la conversión directa de las mediciones de ángulo de progresión en centímetros en la escala de palpación convencional (16).

Otros parámetros indican la estación indirectamente: a) la distancia entre la cabeza y la sínfisis (DCS) que es la distancia entre el borde inferior de la sínfisis del pubis materno y el cráneo fetal, a lo largo de la línea infrapúbica; b) la dirección de la cabeza fetal, que indica la dirección del eje más largo reconocible de la cabeza fetal con respecto al eje largo de la sínfisis púbica (16).

Con una simple rotación en sentido horario del transductor a $90^{\circ}$, se obtiene un plano axial, en el que se pueden evaluar y medir dos parámetros adicionales: la distancia entre la cabeza y el perineo (DCP), como un marcador de la estación principal; y el ángulo de la línea media (ALM), que evalúa la rotación de la cabeza (18).
Para medir la distancia cabeza-perineo, el transductor debe colocarse entre los labios mayores (en la horquilla posterior) y el tejido blando debe comprimirse completamente contra el hueso púbico. El transductor debe estar en ángulo hasta que el contorno del cráneo sea lo más claro posible, lo que indica que el haz de ultrasonido es perpendicular al cráneo fetal. Se mide en una exploración transperineal frontal como la distancia más corta desde el límite óseo externo del cráneo fetal hasta el perineo. Esta distancia representa la parte del canal de parto que aún debe pasar el feto. Varios estudios coinciden en el valor que corresponde a la estación cero, el cual se encuentra entre $35-38 \mathrm{~mm}$ (16).

El ángulo de la línea media difiere de los otros parámetros, ya que utiliza el ángulo de rotación de la cabeza como un indicador del progreso del nacimiento. Se mide en el plano axial mediante un abordaje transperineal: se identifica la línea ecogénica interpuesta entre los dos hemisferios cerebrales (línea media), y el ángulo de la línea media es el ángulo entre esta línea y el eje anteroposterior de la pelvis materna. Se encontró una correlación significativa entre la estación principal evaluada clínicamente y la rotación representada por el ángulo de la línea media (16).

Las indicaciones para el uso del ultrasonido en la evaluación del trabajo de parto se resumen en las siguientes: progreso lento o detención del parto en la primera etapa o la segunda etapa y determinación de la posición de la cabeza fetal antes de considerar o realizar un parto vaginal instrumental $(15,16)$.

El examen digital siempre ha sido el método gold standard para evaluar el descenso de cabeza fetal, la maduración y la dilatación cervical, antes y durante la labor y ha llevado al desarrollo de partogramas integrales utilizados en la mayoría de las instituciones, sin embargo, como se ha mencionado anteriormente, suele ser inconsistente e inexacto, generando angustia en las mujeres y la pérdida de confianza en su 
cuidador o monitorizador del trabajo de parto (2). La evaluación de la dilatación y el borramiento cervical, pueden hacerse por ultrasonido $2 \mathrm{D}$, vía transperineal colocando el transductor a nivel de la horquilla vulvar posterior, en posición sagital, se realizan finos movimientos laterales para obtener imágenes de la sínfisis del pubis materna y del cráneo fetal. La parte anterior del cuello uterino es identificada sobre la parte superior del cráneo fetal en una vista sagital. El transductor es rotado en $90^{\circ}$ sin perder la imagen de la parte superior del cuello uterino o la parte superior del cráneo fetal, se realizan ligeros movimientos del transductor para obtener una visión clara del cuello uterino, hasta obtener el aspecto circular del cuello uterino (2). La medición de la dilatación cervical por ecografía se obtiene en el plano anteroposterior con los cursores en la parte interna del tejido cervical anterior y la parte interna del tejido cervical posterior (interno - interno) $(2,14)$. La apariencia ecográfica del cuello uterino dilatado depende de si las membranas están rotas o no. En los casos en que las membranas no se rompieron, la apariencia ecográfica depende del descenso de la cabeza, que puede mostrar algo de pelo de la cabeza fetal dentro de la luz cervical, o el contenido de líquido amniótico, que también puede mostrar ecos internos en el lumen cervical (14).

La calidad de la visualización cervical por ultrasonido se evalúa en una escala de 0 a 3 para determinar el borramiento cervical de la siguiente manera: (2)

- 3 , donde más del $75 \%$ de la circunferencia cervical es visible

- 2, donde es visible el $50 \%$ - $75 \%$ de la circunferencia cervical

- 1 , donde es visible el $25 \%-50 \%$ de la circunferencia cervical

- 0 , donde se observa menos del $25 \%$ de la circunferencia cervical.
El presente estudio se realizó con el objetivo de determinar la utilidad de la ecografía transperineal intraparto en la progresión del trabajo de parto en las pacientes ingresadas al Servicio de Sala de Partos de la Maternidad Concepción Palacios, en el periodo mayoagosto de 2019.

\section{MÉTODOS}

Se realizó un estudio prospectivo, descriptivo y longitudinal, que incluyó una muestra probabilística, según los siguientes parámetros. Se definió como punto primario de análisis los casos de partos vaginales estimados tanto por ecografía transperineal (ángulo de progresión $>120^{\circ}$ ) y para Bishop ( $>7$ puntos). Así, suponiendo que el $90 \%$ y el 75,3\% de los pares son positivos para ángulo de progresión y para Bishop, respectivamente, la correlación entre la observación emparejada será del $20 \%$ y después de aplicar la corrección de continuidad, el estudio requeriría un tamaño de muestra de 98 pares de pacientes para lograr una potencia del $80 \%$ y una significación de dos lados del $5 \%$ para detectar una diferencia de $-0,143$ entre proporciones marginales (19).

Las pacientes debían ser mayores de 14 años de edad, con gestación única, a término en trabajo de parto, feto en presentación cefálica de vértice, peso fetal estimado mayor a $2500 \mathrm{~g}$ y menor de $4000 \mathrm{~g}$ y con bienestar fetal conservado. Se excluyeron aquellos casos con indicación para cesárea, fetos con circunferencia cefálica $\geq 345 \mathrm{~mm}$, ausencia de latido cardiaco fetal, patologías fetales como restricción del crecimiento intrauterino y malformaciones congénitas, en pacientes con cesárea anterior, aquellas con un segmento uterino $<4,1 \mathrm{~mm}$, y patologías maternas como trastornos hipertensivos del embarazo, cardiopatías, diabetes mellitus y enfermedades renales.

Todas las pacientes firmaron un formulario de consentimiento informado. 
Se les realizó una ecografía transabdominal inicial, con transductor tipo convex de 3,5 MHz, para establecer la biometría fetal y el perfil biofísico, ambos con el fin de determinar el peso estimado fetal y el bienestar fetal; posteriormente se estableció la posición y variedad de posición de la cabeza fetal, en aquellas pacientes que presentaban una cesárea previa, se les realizó la medición del segmento uterino.

Posteriormente se les realizó ecografía transperineal con transductor convex $3,5 \mathrm{mHz}$, cubierto con gel de transmisión y con un guante estéril, para diagnosticar si se encuentra en fase activa del trabajo de parto, mediante la determinación ecográfica de la longitud, dilatación y borramiento cervical, la valoración de la estación y el descenso de la cabeza fetal mediante la determinación del ángulo de progresión o de descenso (AoP), distancia de progresión, estación sonográfica de la cabeza, distancia cabeza-sínfisis, dirección de la cabeza fetal, distancia cabeza-perineo y ángulo de la línea media. La evaluación de la estación y descenso de la cabeza fetal fue realizada cada dos horas, luego de la ecografía inicial, que correspondía a la hora cero. También se practicó tacto vaginal y su descripción por Bishop para correlacionarla con los hallazgos ecográficos. La persona que realizó el tacto vaginal desconocía los hallazgos ecográficos y viceversa.

Al final del parto, se le realizó una pequeña encuesta a la paciente, donde se le preguntaba la tolerancia a la realización de la ecografía intraparto, calificada como satisfecha, indiferente o insatisfecha.

Para evaluar los cambios en el tiempo de las variables ordinales (dilatación, borramiento, variedad de posición, estación de la cabeza y dirección de la cabeza) se aplicó la prueba de homogeneidad marginal, los cambios en las variables ángulo de progresión, distancia cabeza-sínfisis, distancia cabeza-periné y ángulo de línea media, se evaluaron con un modelo líneogeneral de medidas repetidas. Las diferencias entre dilatación (en centímetros) por ecografía vs tacto vaginal, se realizaron con la prueba $\mathrm{T}$ de Student pareada. Las diferencias de borramiento por tacto vaginal con valores medio de borramiento por ecografía se llevaron a cabo con un modelo de análisis de varianza de una vía, el mismo procedimiento se aplicó para verificar diferencias de ángulo de progresión según planos de Hodge. La correlación de estación de la cabeza respecto a distancia cabezasínfisis y cabeza-periné, se llevó a cabo con coeficiente de correlación de Pearson. Se consideró un valor como estadísticamente significativo si $\mathrm{p}<0,05$. Los datos fueron tabulados con JASP versión 0.11 para Windows.

\section{RESULTADOS}

Se evaluó un total de 98 pacientes en trabajo de parto, a las que se les realizó ecografía transperineal intraparto al ingreso (hora 0 ) y a la hora 2. A la hora 4 se realizó el segundo control (23 gestantes), habiéndose realizado un total de 219 ecografías.

En la tabla 1 se representa la distribución de pacientes según las características del cuello durante el trabajo de parto. Al ingreso, 60 pacientes $(61,2 \%)$ tenían dilatación entre 3 y $6 \mathrm{~cm}$, a las 2 horas, 56 pacientes

Tabla 1. Características ecográficas de cuello durante la progresión del trabajo de parto

\begin{tabular}{ccccc}
\hline Característica & $\begin{array}{c}\text { Hora 0 } \\
\mathrm{n}(\%)\end{array}$ & $\begin{array}{c}\text { Hora 2 } \\
\mathrm{n}(\%)\end{array}$ & $\begin{array}{c}\text { Hora 4 } \\
\mathrm{n}(\%)\end{array}$ & $\mathrm{p}$ \\
\hline Dilatación (cm) & & & & 0,001 \\
$3-4$ & $19(19,4)$ & $4(4,1)$ & $0(0)$ & \\
$5-6$ & $41(41,8)$ & $19(19,4)$ & $4(17,4)$ & \\
$7-8$ & $32(32,7)$ & $19(19,4)$ & $8(34,8)$ & \\
$9-10$ & $6(6,1)$ & $56(57,1)$ & $11(47,8)$ & \\
Borramiento & & & & 0,001 \\
Grado 3 & $4(4,1)$ & $0(0)$ & $0(0)$ & \\
Grado 2 & $13(13,3)$ & $4(4,1)$ & $1(4,3)$ & \\
Grado 1 & $51(52,0)$ & $25(25,5)$ & $5(21,7)$ & \\
Grado 0 & $30(30,6)$ & $69(70,4)$ & $17(73,9)$ & \\
\hline
\end{tabular}


(57,1\%) tenían dilatación de $9-10 \mathrm{~cm}$ y en el tercer control, 19 pacientes $(82,6 \%)$ tenían entre 7 y 10 $\mathrm{cm}, 75$ pacientes ya habían completado el periodo expulsivo $(\mathrm{p}=0,001)$. En cuanto al borramiento, al ingreso, 30 pacientes $(30,6 \%)$ tenían grado 0 y 51 grado $1(52 \%)$. A las dos horas, el borramiento fue catalogado como grado 0 en 69 casos $(70,4 \%)$ y a la hora 4, $17(73,9 \%)$ tenían grado $0(\mathrm{p}=0,001)$.

Se observó que la variedad de posición estuvo entre $\geq 02: 30$ horas $\mathrm{y} \leq 03: 30$ horas, en 50 mujeres $(51 \%)$ a la hora 0 , en $69(70,4 \%)$ a la hora 2 y en $17(73,9 \%)$ a la hora $4(\mathrm{p}=0,033)$. El resto de la distribución se puede observar en la tabla 2.

Tabla 2. Cambios en la variedad de posición durante la progresión del trabajo de parto

\begin{tabular}{lcccc}
\hline Característica & $\begin{array}{c}\text { Hora } 0 \\
\mathrm{n}(\%)\end{array}$ & $\begin{array}{c}\text { Hora } 2 \\
\mathrm{n}(\%)\end{array}$ & $\begin{array}{c}\text { Hora } 4 \\
\mathrm{n}(\%)\end{array}$ & $\mathrm{p}$ \\
\hline $\begin{array}{l}\text { Variedad de } \\
\text { posición }\end{array}$ & & & & 0,033 \\
$\geq 02: 30$ horas y & $50(51)$ & $69(70,4)$ & $17(73,9)$ & \\
$\leq 03: 30$ horas & & & & \\
$\geq 08: 30$ horas y & $19(19,4)$ & $17(17,3)$ & $4(17,4)$ \\
$\begin{array}{l}\leq 09: 30 \text { horas } \\
>03: 30 \text { horas y }\end{array}$ & $18(18,4)$ & $6(6,1)$ & $2(8,7)$ \\
$<08: 30$ horas & & & & \\
$>09: 30$ horas & $11(11,2)$ & $6(6,1)$ & $0(0)$ & \\
$\mathrm{y}<02: 30$ horas & & &
\end{tabular}

Los cambios de la presentación fetal durante la evolución del trabajo de parto, evaluados ecográficamente, se presentan en la tabla 3. El ángulo de progresión de la cabeza fetal estuvo entre 91 y 102 grados en 28 gestantes $(28,6 \%)$ al inicio, $14(14,3 \%)$ a las dos horas y $3(13 \%)$ a las 4 horas. En el otro extremo, fue mayor de 127 grados en 2 (2\%) mujeres al inicio, $11(11,2 \%)$ a las dos horas y 5 a las 4 horas $(15,7 \%)(p=0,002)$.

La distancia cabeza-sínfisis midió entre 0 y $10 \mathrm{~mm}$ en 48 gestantes $(49 \%)$ al inicio, $81(82,7)$ a la hora 2 y 22 $(95,7 \%)$ a la hora $4(\mathrm{p}=0,001)$.
Tabla 3. Cambios en el descenso de la cabeza fetal durante la progresión del trabajo de parto

\begin{tabular}{lcccc}
\hline $\begin{array}{l}\text { Característica del } \\
\text { descenso }\end{array}$ & Hora 0 & Hora 2 & Hora 4 & p \\
\hline $\begin{array}{l}\text { Ángulo de progresión } \\
91-102^{\circ}\end{array}$ & $28(28,6)$ & $14(14,3)$ & $3(13)$ & 0,002 \\
$103-114^{\circ}$ & $38(38,8)$ & $41(41,8)$ & $8(34,8)$ & \\
$115-126^{\circ}$ & $30(30,6)$ & $32(32,7)$ & $7(30,4)$ \\
$127-138^{\circ}$ & $2(2)$ & $9(9,2)$ & $2(2,7)$ \\
$129-150^{\circ}$ & $0(0)$ & $2(2,0)$ & $3(13)$ &
\end{tabular}

Distancia cabeza-sínfisis

$\begin{array}{lccc}0-10 \mathrm{~mm} & 48(49) & 81(82,7) & 22(95,7) \\ 11-20 \mathrm{~mm} & 46(46,9) & 17(17,3) & 1(4,3) \\ 21-30 \mathrm{~mm} & 4(4,1) & 0(0) & 0(0)\end{array}$

Estación de la cabeza

$-4,-3$

$88(89,8) \quad 72(73,5) \quad 14(60,9)$

$-2$

$6(6,1) \quad 11(11,2) \quad 4(17,4)$

$-1$

0

$4(1,0) \quad 10(10,2) \quad 0(0)$

$+1,+2,+3$

$0(0) \quad 3(3,1) \quad 3(13)$

$0(0) \quad 2(2,0) \quad 2(8,7)$

Dirección de la cabeza

Abajo

$28(28,6) \quad 34(34,7) \quad 10(43,5)$

Horizontal

$22(22,4) \quad 13(13,3) \quad 4(17,4)$

Arriba

$48(49,0) \quad 51(52,0) \quad 9(39,1)$

Distancia cabeza-periné

0,001
$0-10 \mathrm{~mm}$
$3(3,1) \quad 19(19,4) \quad 9(39,1)$
$11-20 \mathrm{~mm}$
$41(41,8) \quad 52(53,1) \quad 12(52,2)$
$21-30 \mathrm{~mm}$
$39(39,8) \quad 23(23,5) \quad 1(4,3)$
$31-40 \mathrm{~mm}$
$12(12,2) \quad 3(3,1) \quad 1(4,3)$
$41-50 \mathrm{~mm}$
$3(3,1)$
1 (1)
$0(0)$

Ángulo de la línea media

$0-20^{\circ}$

$0(0) \quad 0(0) \quad 2(8,7)$

$21-40^{\circ}$

$5(5,1) \quad 8(8,2) \quad 3(13,0)$

$41-60^{\circ}$

$20(20,4) \quad 43(43,9) \quad 6(26,1)$

$61-80^{\circ}$

$44(44,9) \quad 30(30,6) \quad 10(43,5)$

$81-100^{\circ}$

$29(29,6) \quad 17(17,3) \quad 2(8,7)$ 
La estación de la cabeza era $-4,-3$ en $88(89,8 \%)$ pacientes al inicio, $72(73,5 \%)$ a la hora 2 y $14(60,9 \%)$ a la hora 4. Por el contrario, no hubo pacientes con la presentación en estación $0,+1,+2,+3$ al ingreso. A la hora 2 habían $5(5,1 \%)$ pacientes y a la hora 4,5 pacientes $(21,7 \%)(\mathrm{p}=0,001)$.

La distancia cabeza-periné fue de $20 \mathrm{~mm}$ o menos en 44 pacientes $(44,9 \%)$ a la primera evaluación, en 71 gestantes $(72,4 \%)$ a la hora 2 y en 21 a la hora 4 $(91,3 \%)(\mathrm{p}=0,001)$.

El ángulo de la línea media fue de mayor a 40 grados en 93 casos a la hora 0 (94,9\%), en $90(91,8 \%)$ a la hora $2 \mathrm{y}$ en $18(78,3 \%)$ a la hora $4(\mathrm{p}=0,001)$.

Se comparó la dilatación clínica obtenida a través del tacto vaginal con respecto a la obtenida por ecografía transperineal, expresadas en medias y desviación estándar (tabla 4). Al ingreso, la media ecográfica fue de $5,94 \pm 1,70 \mathrm{~cm}$ y la media por tacto fue 5,96 $\pm 1,80 \mathrm{~cm}(\mathrm{p}=0,717)$. A las dos horas, los valores medios fueron $8,14 \pm 1,92 \mathrm{~cm}$ y $8,15 \pm 2,07 \mathrm{~cm}$, respectivamente $(\mathrm{p}=0,849)$. Al tercer control, hora 4 , la media obtenida por ecografía fue $8,35 \pm 1,70 \mathrm{~cm}$ y por tacto fue $8,43 \pm 1,97(\mathrm{p}=0,604)$.

Se correlacionó el borramiento obtenido en las 219 ecografías con el obtenida en los 219 tactos, independientemente de la hora del tacto y se representa en la tabla 5. Entre las 4 pacientes diagnosticadas ecográficamente como grado 3 (el

Tabla 4. Comparación de dilatación (en cm) entre ecografía y tacto vaginal

\begin{tabular}{lcccc}
\hline Tiempo & $\mathrm{n}$ & Ecografía & Tacto vaginal & $\mathrm{p}$ \\
\hline 0 horas & 98 & $5,94 \pm 1,70$ & $5,96 \pm 1,80$ & 0,717 \\
2 horas & 98 & $8,14 \pm 1,92$ & $8,15 \pm 2,07$ & 0,849 \\
4 horas & 23 & $8,35 \pm 1,70$ & $8,43 \pm 1,97$ & 0,604 \\
\hline
\end{tabular}

Tabla 5. Distribución comparativa de pacientes según el borramiento por ecografía y por tacto vaginal

\begin{tabular}{lccc}
\hline \multirow{3}{*}{ Ecografía } & \multicolumn{3}{c}{ Tacto vaginal } \\
\cline { 2 - 4 } & $\begin{array}{c}40 \%-50 \% \\
\mathrm{n}(\%)\end{array}$ & $\begin{array}{c}60 \%-70 \% \\
\mathrm{n}(\%)\end{array}$ & $\begin{array}{c}>80 \% \\
\mathrm{n}(\%)\end{array}$ \\
\hline Grado 3 & $1(25)$ & $3(75)$ & 0 \\
Grado 2 & $6(33,3)$ & $9(50)$ & $3(16,7)$ \\
Grado 1 & 0 & $24(29,6)$ & $57(70,4)$ \\
Grado 0 & 0 & 0 & $116(100)$ \\
\hline$(\mathrm{p}=0,002)$ & & &
\end{tabular}

menor borramiento), 1 (25\%) tenía cuello borrado $40 \%$ - 50 \% y 3 (75 \%), $60 \%$ - $70 \%$. Las $116(100 \%)$ pacientes diagnosticadas ecográficamente como grado 0 (el máximo de borramiento), tenían cuello borrado más de $80 \%$. La correlación fue significativa $(\mathrm{p}=0,002)$.

También se relacionó el descenso de la cabeza fetal por tacto (planos de Hodge) con los parámetros ecográficos: distancia cabeza-sínfisis (DCS), distancia cabeza-periné (DCP), ángulo de la línea media (ALM) y ángulo de progresión. Estos datos se presentan en la tabla 6 .

Había 153 ecografías de pacientes con DCS entre 0 y $10 \mathrm{~mm} ; 113(73,9 \%)$ pacientes estaban en primer plano, $30(19,6 \%)$ en segundo plano, 6 (3,9\%) en tercer plano y $4(2,6 \%)$ en cuarto plano. Hubo 62 casos con DCS entre 11 y $20 \mathrm{~mm}, 57$ (91,9\%) estaban en primer plano y $5(8,1 \%)$ en segundo plano $(\mathrm{p}=0,124)$.

La DCP fue de 11 a $20 \mathrm{~mm}$ en 105 ecografías. En 77 $(73,3 \%)$ la presentación estaba en primer plano, en 26 $(24,8 \%)$ estaba en segundo plano y en $2(1,9 \%)$ en tercer plano; en 20 ecografías, la DCP era mayor de 30 $\mathrm{mm}$, todos estaban en primer plano $(\mathrm{p}=0,001)$.

El ALM fue 61-80 grados en 84 estudios. En 72 $(85,7 \%)$, la presentación estaba en primer plano, 9 $(10,7 \%)$ en segundo, $1(1,2 \%)$ en tercero y $2(2,4 \%)$ en 4 plano $(p=0,001)$. 
Tabla 6. Relación entre nivel de la cabeza fetal por tacto vaginal y por ecografía

\begin{tabular}{|c|c|c|c|c|c|}
\hline \multirow{2}{*}{ Ecografía } & \multicolumn{4}{|c|}{ Tacto vaginal } & \multirow{2}{*}{$\mathrm{p}$} \\
\hline & 1 plano $\mathrm{n}(\%)$ & 2 plano n $(\%)$ & 3 plano $\mathrm{n}(\%)$ & 4 plano $\mathrm{n}(\%)$ & \\
\hline Distancia cabeza-sínfisis & & & & & 0,124 \\
\hline $0-10 \mathrm{~mm}$ & $113(73,9)$ & $30(19,6)$ & $6(3,9)$ & $4(2,6)$ & \\
\hline $11-20 \mathrm{~mm}$ & $57(91,9)$ & $5(8,1)$ & --- & --- & \\
\hline $21-30 \mathrm{~mm}$ & $3(75)$ & $1(25)$ & --- & --- & \\
\hline Distancia cabeza-periné & & & & & 0,001 \\
\hline $0-10 \mathrm{~mm}$ & $15(48,4)$ & $8(25,8)$ & $4(12,9)$ & $4(12,9)$ & \\
\hline $11-20 \mathrm{~mm}$ & $77(73,3)$ & $26(24,8)$ & $2(1,9)$ & --- & \\
\hline $21-30 \mathrm{~mm}$ & $61(96,8)$ & $2(3,2)$ & --- & --- & \\
\hline $31-40 \mathrm{~mm}$ & $16(100)$ & --- & --- & --- & \\
\hline $41-50 \mathrm{~mm}$ & $4(100)$ & --- & --- & --- & \\
\hline Ángulo de la línea media & & & & & 0,001 \\
\hline $0-20^{\circ}$ & --- & --- & $2(100)$ & --- & \\
\hline $21-40^{\circ}$ & $10(62,5)$ & $4(25)$ & --- & $2(12,5)$ & \\
\hline $41-60^{\circ}$ & $48(69,6)$ & $18(26,1)$ & $3(4,3)$ & --- & \\
\hline $61-80^{\circ}$ & $72(85,7)$ & $9(10,7)$ & $1(1,2)$ & $2(2,4)$ & \\
\hline $81-100^{\circ}$ & $43(89,6)$ & $5(10,4)$ & --- & --- & \\
\hline Ángulo de progresión & & & & & 0,001 \\
\hline $91-102^{\circ}$ & $44(97,8)$ & $1(2,2)$ & --- & --- & \\
\hline $103-114^{\circ}$ & $87(100)$ & -- & --- & --- & \\
\hline $115-126^{\circ}$ & $42(60,9)$ & $27(39,1)$ & --- & --- & \\
\hline $127-138^{\circ}$ & --- & $8(61,5)$ & $5(38,5)$ & --- & \\
\hline $139-150^{\circ}$ & --- & --- & $1(20)$ & $4(80)$ & \\
\hline
\end{tabular}

Con respecto al ángulo de progresión, los 87 estudios con ángulo de progresión de 103 a $114,{ }^{\circ}$ estaban en primer plano. Los 5 casos con ángulo de progresión de $139-150,{ }^{\circ}$ estaban 1 , en tercer plano y 4 en cuarto plano $(\mathrm{p}=0,001)$.

En la tabla 7 se presenta el coeficiente de correlación de Spearman entre la estación de la cabeza por clínica y las distancias cabeza-sínfisis y cabeza-periné. En relación con la DCS, se distribuyó entre $-0,302$ a la hora 2 y $-0,622$ a la hora 4 . En relación con la DCP, el coeficiente estuvo entre $-0,429$ y $-0,524$.

Todas las pacientes toleraron el seguimiento del trabajo de parto con ecografía y expresaron su satisfacción.
Tabla 7. Coeficiente de correlación de Spearman entre estación vs distancia cabeza-sínfisis y distancia cabeza-periné

\begin{tabular}{lccc}
\hline Tiempo & Variables & $\mathrm{R}$ & $\mathrm{p}$ \\
\hline 0 horas & Estación vs DCS* & $-0,325$ & 0,001 \\
& Estación vs DCP** & $-0,440$ & 0,001 \\
\multirow{2}{*}{2 horas } & Estación vs DCS* & $-0,302$ & 0,001 \\
& Estación vs DCP** & $-0,429$ & 0,001 \\
\multirow{3}{*}{4 horas } & Estación vs DCS* & $-0,622$ & 0,001 \\
& Estación vs DCP** & $-0,524$ & 0,001 \\
\hline
\end{tabular}

*Distancia cabeza-sínfisis

**Distancia cabeza periné 


\section{DISCUSIÓN}

El parto es el proceso fisiológico que pone fin al embarazo, por el cual se produce la salida desde el útero hacia el exterior a través del canal del parto, de un feto, vivo o muerto, seguido de la placenta y de las membranas ovulares; a lo largo del tiempo se ha realizado el seguimiento del trabajo de parto a través del tacto vaginal (1). En la presente investigación, se realizó el seguimiento de la progresión del trabajo de parto a través de la ecografía intraparto, intentando demostrar su utilidad para evitar la subjetividad de la evaluación clínica, además de la incomodidad que genera en la paciente dicha evaluación.

Se evaluaron parámetros ecográficos que tuvieran correlación clínica en la evaluación de la progresión del trabajo de parto, con la finalidad de realizar procedimientos menos invasivos y más precisos. Se realizaron comparaciones de la dilatación y borramiento ecográfico vs los hallazgos obtenidos a través del tacto vaginal (patrón estándar), según los parámetros establecidos en el trabajo de Hassan y cols. (2) en la evaluación 2D de la circunferencia cervical, para determinar la dilatación y borramiento del cuello, adicional a esto, se utilizaron variables establecidas por los lineamientos de la ISOUG (16) en la ecografía intraparto con respecto al descenso de la calota fetal en el canal del parto, tales como ángulo de progresión (AoP), estación ecográfica de la cabeza, dirección de la cabeza, distancia cabeza-sínfisis (DCS), ángulo de línea media y distancia cabeza-periné (DCP).

Se realizó ecografía transperineal a 98 pacientes, en trabajo de parto, con rango de edades entre 16-43 años, realizando 2 o 3 evaluaciones, según la dilatación inicial. Se incluyeron en el estudio pacientes primigestas, multíparas y/o con una cesárea anterior; inicialmente se diagnosticó, mediante la evaluación digital (tacto vaginal), que la paciente se encontraba en fase activa del trabajo de parto $(3-10 \mathrm{~cm})$, documentando la dilatación en centímetros y el borramiento en porcentaje; posteriormente se realizó la ecografía transperineal y se evaluó la dilatación (diámetro antero-posterior) en centímetros y el borramiento cervical (visualización de la circunferencia cervical) en grados, evidenciando que, los cambios detectados en la evaluación clínica a través del tacto, son perfectamente evaluables a través de la ecografía transperineal; no se demostró diferencia estadísticamente significativa entre ambos procedimientos para la evaluación de la dilatación cervical, lo que demuestra una concordancia clínicoecográfica y deja en evidencia que la ecografía transperineal es de utilidad en el diagnóstico de los cambios del cuello uterino durante el trabajo de parto, logrando tener una evaluación mucho más objetiva, menos invasiva y dolorosa para la paciente. El estudio arrojó resultados similares a los obtenidos por Hassan y cols. (2) en 2013, quienes evaluaron a 19 mujeres, realizaron una comparación entre la dilatación y borramiento cervical por tacto vaginal vs la obtenida por ecografía transperineal y obtuvieron una correlación fuerte y positiva entre los valores del tacto vaginal y los valores obtenidos por ecografía, adicionalmente demuestran una buena aceptación del procedimiento ecográfico sobre la evaluación digital.

En cuanto a la evaluación del descenso de la cabeza fetal durante el trabajo de parto, se tomaron varias medidas propuestas por la ISOUG (16), tales como AoP, DCS, DCP, dirección de la cabeza y ALM; se evidenció que a medida que avanza el trabajo de parto y la cabeza fetal va descendiendo a través del canal del parto, el AoP obtenido iba aumentando con respecto al tiempo, comportamiento similar al descrito en los trabajos de Ghi y cols. (6) y Tutschek y cols. (5) publicados en 2013, y de Ghi y cols. (16), de la ISOUG; sin embargo, a pesar de dicho hallazgo, se pudo evidenciar que el ángulo arrojado en esta serie fue mucho mayor al obtenido en los trabajos citados. La estación cero corresponde a un ángulo de progresión de $116^{\circ},(5,6,16)$ a diferencia del obtenido en este estudio que correspondía a $131^{\circ}$. 
Con respecto a la evaluación de la DCS y DCP, se observó una fuerza de asociación intensa e inversa, es decir, hubo una disminución de ambas distancias a medida que asciende la estación fetal hacia números positivos, estos cambios fueron estadísticamente significativos, similar a los resultados publicados previamente $(5,16,20)$.

Por último, al igual que los resultados obtenidos por Tutschek y cols. (5), Ghi y cols. (16) y Hassan y cols. (2), se evaluó la tolerancia de las pacientes a la ecografía transperineal intraparto con respecto al tacto vaginal y se evidenció que todas las pacientes evaluadas se sintieron satisfechas con la ecografía, de esta manera se recomienda la ecografía transperineal como la evaluación adecuada para las pacientes en trabajo de parto, ofreciendo mayor comodidad a las mismas.

La ecografía transperineal es un procedimiento sencillo y útil para la evaluación del trabajo de parto, dando parámetros objetivos en la progresión del trabajo de parto, teniendo resultados equiparables a la evaluación clínica, muy bien tolerado por las pacientes, con un $100 \%$ de aceptación sobre el tacto vaginal, por ser un método de evaluación práctico, no invasivo ni doloroso para la paciente.

La ecografía transperineal, posee una excelente correlación clínica en la evaluación de la dilatación y borramiento cervical, por lo que podría implementarse en la práctica habitual, luego de una curva de aprendizaje para el dominio de la técnica. La DCS, DCP y el AoP son parámetros ecográficos útiles para la identificación de la estación de la cabeza fetal mediante ecografía, particularmente en aquellos casos de cabeza moldeada o presencia de caput sucedaneum, que pueden dificultar la interpretación adecuada de la estación de la cabeza en el tacto vaginal.
Se concluye que:

La ecografía transperineal intraparto es útil en la evaluación de la progresión del trabajo de parto, con buena correlación entre el tacto y la ecografía y excelente tolerancia por parte de las pacientes.

Se recomiendan estudios complementarios para validar y reafirmar los hallazgos obtenidos en esta investigación.

Los autores expresan su agradecimiento al licenciado Angulo, por su paciencia en cada cambio realizado durante el procesamiento estadístico, además de su asesoría que contribuyó a lograr un mejor análisis de los hallazgos encontrados. A la Dra. Mireya González Blanco, asesor metodológico, igracias totales! $\mathrm{Su}$ experiencia científica logró dar el toque de precisión y perfección que queríamos lograr.

\section{Sin conflictos de interés.}

\section{REFERENCIAS}

1. González C, Goñi Z, Melchor M. Concepto y definición del parto normal. Causas del inicio del parto. Signos prodrómicos y diagnóstico del parto. En: Bajo Arenas J, Melchor M, Mercé L, editores. Fundamentos de obstetricia (SEGO). Madrid: Editorial Médica Panamericana; 2007. p 339-346.

2. Hassan W, Eggebo T, Ferguson M, Lees C. Simple 2D ultrasound technique to assess intrapartum cervical dilatation: a pilot study. Ultrasound Obstet Gynecol. 2013; 41(4):413-418. doi: 10.1002/uog.12316.

3. Youssef A, Bellussi F, Maroni E, Pilu G, Rizzo N, Ghi T. Ultrasound in labor: is it time for a more simplified approach? Ultrasound Obstet Gynecol. 2013; 41(6):710-711. doi: 10.1002/uog.12373.

4. Kalache K, Duckelmann A, Michaelis S, Lange J, Cichon G, Dudenhausen J. Transperineal ultrasound imaging in prolonged second stage of labor with occipitoanterior presenting fetuses: how well does the 'angle of progression' predict the mode of delivery? Ultrasonido Gynecol Obstet. 2009; 33(3):326-330. doi: 10.1002/uog.6294 


\section{G J CÁRDENAS MENDOZA ET AL.}

5. Tutschek B, Torkildsen E, Eggebo T. Comparison between ultrasound parameters and clinical examination to assess fetal head station in labor. Ultrasound Obstet Gynecol. 2013; 41(4):425-429. doi: 10.1002/ uog. 12422.

6. Ghi T, Youssef A, Maroni E, Arcangely T, Musso T, Bellussi $\mathrm{F}$, et al. Intrapartum transperineal ultrasound assessment of fetal head progression in active second stage of labor and mode of delivery. Ultrasonido Gynecol Obstet. 2013; 41(4):430-435. doi: 10.1002/ uog. 12379.

7. Rodríguez L. Ecografía intraparto: valor como método diagnóstico para determinar la prolongación del tiempo de expulsivo y la vía de parto [Tesis de Grado]. Zaragoza: Universidad de Zaragoza; 2017 [consultado abril 2019]. Disponible en: https://dialnet.unirioja.es/ servlet $/$ tesis? codigo $=202244$

8. Sainz JA, García-Mejido JA, Aquise A, Borrero C, Bonomi MJ, Fernández-Palacín A. A simple model to predict the complicated operative vaginal deliveries using vacuum or forceps. Am J Obstet Gynecol. 2019; 220(2):193.e1-193.e12. doi: 10.1016/j. ajog.2018.10.035.

9. Malvasi A, Tinelli A, Barbera A, Eggebø TM, Mynbaev OA, Bochicchio $\mathrm{M}$, et al. Occiput posterior position diagnosis: vaginal examination or intrapartum sonography? A clinical review. J Matern Fetal Neonatal Med. 2014; 27(5):520-526. doi: 10.3109/14767058.2013.825598.

10. Barbera AF, Pombar X, Perugino G, Lezotte DC, Hobbins JC. A new method to assess fetal head descent in labor with transperineal ultrasound. Ultrasound Obstet Gynecol. 2009; 33(3):313-319. doi: 10.1002/ uog.6329.

11. Eggebø TM, Gjessing LK, Heien C, Smedvig E, Økland I, Romundstad P, et al. Prediction of labor and delivery by transperineal ultrasound in pregnancies with prelabor rupture of membranes at term. Ultrasound Obstet Gynecol. 2006; 27(4):387-391. doi: 10.1002/ uog. 2744.

12. Gilboa Y, Kivilevitch Z, Spira M, Kedem A, Katorza $\mathrm{E}$, Moran $\mathrm{O}$, et al. Pubic arch angle in prolonged second stage of labor: clinical significance. Ultrasound Obstet Gynecol. 2013; 41(4):442-446. doi: 10.1002/ uog. 12304.
13. Mendoza A, Pages A. Ecografía transperineal: índice cervical para la inducción del trabajo de parto [Trabajo Especial de Grado]. Caracas: Universidad Central de Venezuela; 1994.

14. Wiafe YA, Whitehead B, Venables H, Dassah ET, Eggebø TM. Intrapartum ultrasound assessment of cervical dilatation and its value in detecting active labor. J Ultrasound. 2018; 21(3):233-239. doi: 10.1007/ s40477-018-0309-2.

15. Clínic Barcelona. Hospital Universitario [Internet]. Barcelona: Protocolo: valoración ecográfica intraparto; 2011 [consultado abril 2019]. Disponible en: https:// medicinafetalbarcelona.org/protocolos/es/obstetricia/ ecograf\%C3\%ADa\%20intraparto.pdf.

16. Ghi T, Eggebø T, Lees C, Kalache K, Rozenberg $\mathrm{P}$, Youssef A, et al. ISUOG Practice Guidelines: intrapartum ultrasound. Ultrasound Obstet Gynecol. 2018; 52(1):128-139. doi: 10.1002/uog.19072.

17. Yeo L, Romero R. Sonographic evaluation in the second stage of labor to improve the assessment of labor progress and its outcome. Ultrasound Obstet Gynecol. 2009; 33(3):253-258. doi: 10.1002/uog.6336.

18. Montesinos L, Merino M, Melchor M. Mecanismo del parto. El motor del parto: el objeto del parto. En: Bajo Arenas J, Melchor M, Mercé L, editores. Fundamentos de obstetricia (SEGO). Madrid: Editorial Médica Panamericana; 2007. p 347-350.

19. Connor RJ. Sample size for testing differences in proportions for the paired-sample design. Biometrics [Internet]. 1987 [consultado abril 2019]; 43(1):207-211. Disponible en: https://www.jstor.org/stable/2531961

20. Hassan W, Eggebo T, Ferguson M, Gillett A, Studd J, Pasupathy D, et al. The sonopartogram: a novel method for recording progress of labor by ultrasound. Ultrasound Obstet Gynecol. 2014; 43 (2): 189-194. doi: 10.1002/uog. 13212. 